\title{
Electron-Electron Scattering in a Double Quantum Dot: Effective Mass Approach
}

S. Yu. Kruchinin

Saint Petersburgh State University of Information Technologies, Mechanics and Optics

A. V. Federov

Saint Petersburg State University of Information Technologies, Mechanics and Optics

A. V. Baranov

Saint Petersburgh State University of Information Technologies, Mechanics and Optics

See next page for additional authors

Follow this and additional works at: https://arrow.tudublin.ie/engscheceart

Part of the Electrical and Electronics Commons

\section{Recommended Citation}

Kruchinin, S.Yu., Fedorov, A.V., Baranov, A.V., Perova, T.S. and Kevin Berwick : Electron-electron scattering in a double quantum dot: Effective mass approach. Journal of Chemical Physics, 133, 10, 2010, 104704. doi:10.1063/1.3477766

This Article is brought to you for free and open access by the School of Electrical and Electronic Engineering at ARROW@TU Dublin. It has been accepted for inclusion in Articles by an authorized administrator of ARROW@TU Dublin. For more information, please contact arrow.admin@tudublin.ie, aisling.coyne@tudublin.ie, gerard.connolly@tudublin.ie.

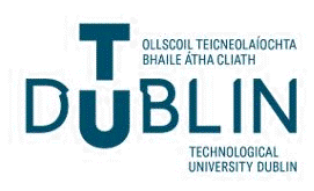




\section{Authors}

S. Yu. Kruchinin, A. V. Federov, A. V. Baranov, Tatiana Perova, and Kevin Berwick

This article is available at ARROW@TU Dublin: https://arrow.tudublin.ie/engscheceart/135 


\title{
Electron-electron scattering in a double quantum dot: Effective mass approach
}

\author{
S. Yu. Kruchinin, ${ }^{1, a)}$ A. V. Fedorov, ${ }^{1, b)}$ A. V. Baranov, ${ }^{1}$ T. S. Perova ${ }^{2}$ and K. Berwick ${ }^{3}$ \\ ${ }^{1}$ Saint-Petersburg State University of Information Technologies, Mechanics and Optics, \\ 49 Kronverksky Avenue, 197101 St. Petersburg, Russia \\ ${ }^{2}$ Department of Electronic and Electrical Engineering, Trinity College, University of Dublin, Dublin 2, \\ Ireland \\ ${ }^{3}$ Department of Electronic and Communications Engineering, Dublin Institute of Technology, Dublin 8, \\ Ireland
}

(Received 10 June 2010; accepted 20 July 2010; published online 10 September 2010)

\begin{abstract}
We present a theoretical description of the first-order scattering of interacting electrons and holes in a double quantum dot. Assuming infinitely high walls, strong confinement, and a two-band approximation, we derive general expressions for the two-particle matrix elements of the screened Coulomb potential. We also determine the selection rules for different scattering channels and consider special cases where the corresponding matrix elements can be represented by simple analytical expressions. Numerical calculations of the matrix elements and an analysis of their dependence on the geometrical and material parameters of the double quantum dot have also been performed. (C) 2010 American Institute of Physics. [doi:10.1063/1.3477766]
\end{abstract}

\section{INTRODUCTION}

Semiconductor nanocrystals, or the so-called quantum dots, with the characteristic linear size $R_{0}$ comparable to the exciton Bohr radius $r_{B}$ of the corresponding bulk crystal, are of great interest because of unusual electronic structure and optical properties. ${ }^{1}$ Depending on a relative magnitude between $R_{0}$ and $r_{B}$, they may be classified into two regimes: for $R_{0} \gg r_{B}$, a weak-confinement regime where the exciton center-of-mass motion is quantized, and for $R_{0} \ll r_{B}$, a strong confinement one where the motions of the electron and the hole are separately quantized. In principle, due to the sizeconfinement effect in quantum dots, the energy spectra of quasiparticles, such as electrons, holes, phonons, and excitons, are transformed. Moreover, the confinement also modifies the interactions between the quasiparticles and external field and between the quasiparticles themselves. In this connection, studies of such interactions, together with the inherent electronic structure, are of considerable importance. In addition, the systems containing quantum dots are expected to be promising materials for some applications in optoelectronics.

Resonant energy transfer due to Coulomb interaction in ordered structures of quantum dots (QDs) has received much attention in recent theoretical ${ }^{2-6}$ and experimental ${ }^{7-9}$ investi- $^{-}$ gations. This phenomenon has been studied using several different approaches, including tight-binding ${ }^{2}$ and pseudopotential $^{5}$ calculations, a quantum-electrodynamical approach $^{3,10}$ and using an effective mass approximation. ${ }^{4,6}$ Previous theoretical treatments have been primarily directed to the investigation of resonant energy transfer involving interband transitions, although variations in the size and mate-

\footnotetext{
${ }^{a)}$ Electronic mail: stanislav.kruchinin@gmail.com.

b)Electronic mail: a_v_fedorov@inbox.ru.
}

rial used in creating the QDs can enable resonance between the states in the electron-hole pair and a single charge carrier, as well as between electron or hole states. Thus, interdot energy transfer processes accompanying the intraband transitions can be induced by Coulomb coupling. A simple analysis of the electron-electron interaction Hamiltonian for a double QD shows that these processes do exist and that they will manifest themselves through the modification of the optical properties of the double QD. For the incoherent energy transfer regime, ${ }^{6,11}$ these processes result in line broadening and an intensity change in the optical spectra, e.g., either an increase or quenching of the luminescence. In the coherent energy transfer regime, ${ }^{12}$ where the transfer rate overcomes the phase relaxation rate, the processes can lead to the appearance of fine structure in the optical spectra due to the quantum entanglement effect. ${ }^{13}$

Previous theoretical and experimental studies confirm the validity of the simple dipole-dipole approximation and use an effective screening constant for the calculation of the two-particle matrix elements of Coulomb interaction in spherical direct-band semiconductor QDs (e.g., Refs. 2, 5, and 7). For instance, the difference between squared matrix elements calculated with the point dipole approximation and that using a pseudopotential calculation is less than $3 \%$ even at quasicontact interdot distances. ${ }^{5}$ Thus, one can expect that the problem symmetry will allow simple expressions describing interdot energy transfer processes accompanied by intraband transitions to be obtained. Doing so will allow the theory of electron-electron interactions for QD systems using a simple, two-band effective mass approximation to be developed.

In this paper, we present the theory of first-order scattering of charge carriers in a double QD. We perform our calculations under the following assumptions: electrons and holes are strongly confined in QDs with infinitely high po- 
tential walls, the interaction of electrons and holes in adjacent QDs is described by the Coulomb potential with an effective screening constant, and the energy spectrum of the charge carriers is described by a two-band effective mass approximation. These simplifications allow us to derive general expressions for the matrix elements that define the probability amplitudes for all significant two-particle processes. In Sec. II, we obtain expressions for the matrix elements of the electrostatic interaction potential. In addition, we discuss the selection rules defining various specific scattering channels. In Sec. III, we provide the results of numerical calculations of the matrix elements and their dependence on the geometric and material parameters for a double quantum dot. A short summary and concluding remarks are given in Sec. IV.

\section{COULOMB MATRIX ELEMENTS IN A DOUBLE QD SYSTEM}

The Hamiltonian for direct electron-electron interaction in a double QD with infinitely high potential walls can be written as

$$
H_{\text {int }}=\iint d^{3} r_{\mathrm{I}} d^{3} r_{\mathrm{II}} \psi_{\mathrm{I}}^{\dagger}\left(\mathbf{r}_{\mathrm{I}}\right) \psi_{\mathrm{II}}^{\dagger}\left(\mathbf{r}_{\mathrm{II}}\right) V\left(\mathbf{r}_{\mathrm{I}}, \mathbf{r}_{\mathrm{II}}\right) \psi_{\mathrm{I}}\left(\mathbf{r}_{\mathrm{I}}\right) \psi_{\mathrm{II}}\left(\mathbf{r}_{\mathrm{II}}\right)
$$

Here, the integration is performed over the volume of the quantum dots, $V\left(\mathbf{r}_{\mathrm{I}}, \mathbf{r}_{\mathrm{II}}\right)$ is the potential of the electronelectron interaction, the coordinates of the electrons $\mathbf{r}_{\alpha}(\alpha$ $=\mathrm{I}$, II) belong to the corresponding QDs, numbered I and II, and $\psi_{\alpha}$ and $\psi_{\beta}^{\dagger}$ are field operators, which can be represented as the sum of the electron $(c)$ and hole $(v)$ components

$$
\begin{aligned}
& \psi_{\alpha}^{\dagger}\left(\mathbf{r}_{\alpha}\right)=\psi_{\alpha, c}^{\dagger}\left(\mathbf{r}_{\alpha}\right)+\psi_{\alpha, v}\left(\mathbf{r}_{\alpha}\right), \\
& \psi_{\alpha, c}^{\dagger}=\sum_{k} a_{k}^{\dagger} \Psi_{k}^{*}\left(\mathbf{r}_{\alpha}\right), \quad \psi_{\alpha, v}=\sum_{k} d_{k} \Psi_{k}\left(\mathbf{r}_{\alpha}\right) .
\end{aligned}
$$

Here $\left(a_{k}, a_{k}^{\dagger}\right)$ and $\left(d_{k}, d_{k}^{\dagger}\right)$ are the creation and annihilation operators for electrons and holes, respectively, and $\Psi_{k}\left(\mathbf{r}_{\alpha}\right)$ are the electron and hole wave functions. Substitution of Eq. (2) into Eq. (1) and rewriting Eq. (1) using normal-ordered products of field operators $^{14,15}$ give the sum of terms that describe a variety of processes, viz., scattering of electrons and holes, creation and annihilation of electron-hole pairs, forward scattering, and others. We do not consider the exchange interaction since it is negligibly small in the QDmatrix systems ${ }^{16}$ studied. Real scattering processes can be represented by the diagrams depicted on Fig. 1.

We assume that the electrostatic interaction of two spherical QDs can be described by the screened Coulomb potential

$$
V\left(\mathbf{r}_{\mathrm{I}}, \mathbf{r}_{\mathrm{II}}\right)=\frac{e^{2}}{\varepsilon\left|\mathbf{r}+\mathbf{r}_{\mathrm{I}}-\mathbf{r}_{\mathrm{II}}\right|} .
$$

Here, $\mathbf{r}$ is the vector directed from the center of QD II to the center of QD I, vectors $\mathbf{r}_{\mathrm{I}}$ and $\mathbf{r}_{\mathrm{II}}$ originate from the center of the corresponding QD, and dielectric screening is taken into account by the effective dielectric constant $\varepsilon$. In general, $\varepsilon$ should be treated as a fitting parameter associated with the

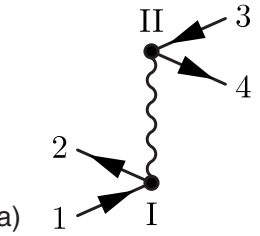

(b)

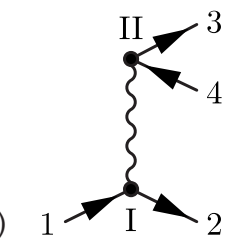

(c)
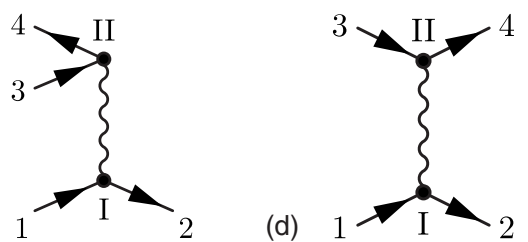

FIG. 1. Sample diagrams describing real first-order processes. Arrows from left to right denote electrons; those from right to left denote holes. Roman numerals denote quantum dots and Arabic numbers denote electron or hole states. (a) Annihilation scattering of two electron-hole pairs (interbandinterband transitions). A second diagram of this type is obtained by swapping the numerals I and II. Scattering with creation (b) and annihilation (c) of one electron-hole pair (interband-intraband transitions). Further, six diagrams of this type can be obtained by swapping the numerals I and II and by changing the directions of the arrows. (d) Processes without creation or annihilation of electron-hole pairs (intraband-intraband transitions). Three other diagrams of this type can be obtained by changing the directions of the arrows.

dielectric constant of the matrix $\varepsilon_{M}$ and the quantum dots, $\varepsilon_{\mathrm{I}}$ and $\varepsilon_{\mathrm{II}}$. In the following analysis, it should be understood that the dielectric constants of the materials should be replaced by the squares of their refractive indices.

Approximate expressions for $\varepsilon$ can be derived as follows. The dielectric screening of the $l_{\mathrm{I}}$ th and $l_{\mathrm{II}}$ th multipole terms generated by interaction between the charge distributions within the spherical QDs I and II is given by the following expression: ${ }^{4,17,18}$

$$
\varepsilon_{l_{\mathrm{I}} l_{\mathrm{II}}}=\frac{\varepsilon_{M}}{f_{l_{\mathrm{I}}}\left(\varepsilon_{\mathrm{I}} / \varepsilon_{M}\right) f_{l_{\mathrm{II}}}\left(\varepsilon_{\mathrm{II}} / \varepsilon_{M}\right)} .
$$

Here, $\varepsilon_{\alpha}$ and $\varepsilon_{M}$ are the dielectric constants of quantum dot $\alpha$ and the matrix, respectively, $l_{\mathrm{I}}$ and $l_{\mathrm{II}}$ are the multipole moments, and

$$
f_{l}\left(\varepsilon_{\alpha} / \varepsilon_{M}\right)=\frac{2 l+1}{\left(\varepsilon_{\alpha} / \varepsilon_{M}+1\right) l+1}
$$

is a field factor describing the screening of the $l$ th pole by the QD material and the matrix. ${ }^{4,17,18}$ A consideration of $\varepsilon_{l_{\mathrm{I}} l_{\mathrm{II}}}$ as a function of $l_{\mathrm{I}}$ and $l_{\mathrm{II}}$, for typical values of the dielectric constants $\varepsilon_{\alpha}$ and $\varepsilon_{M}$, shows that it varies slowly with $l_{\mathrm{II}}$ for fixed values of $l_{\mathrm{I}}$ and vice versa (see Appendix for details). This property of $\varepsilon_{l_{\mathrm{I}}, l_{\mathrm{II}}}$ allows us to use a single effective dielectric constant $\varepsilon=\varepsilon_{l_{\mathrm{I}}^{\prime}, l_{\mathrm{II}}^{\prime}}$, where $l_{\mathrm{I}}^{\prime}$ and $l_{\mathrm{II}}^{\prime}$ are the moments of the multipole term, which provide the main contribution to the electrostatic interdot interaction. In particular, for interbandinterband dipole-allowed transitions, the main term in the multipole expansion corresponds to dipole-dipole interaction, so we have the moments $l_{\mathrm{I}}^{\prime}=1$ and $l_{\mathrm{II}}^{\prime}=1$, and the effective dielectric constant should be taken as 


$$
\varepsilon \equiv \varepsilon_{11}=\frac{\left(\varepsilon_{\mathrm{I}}+2 \varepsilon_{M}\right)\left(\varepsilon_{\mathrm{II}}+2 \varepsilon_{M}\right)}{9 \varepsilon_{M}} .
$$

If the interband transition in the first QD is dipole-allowed but in the second QD is dipole-forbidden, the leading term will correspond to dipole-quadrupole interaction, and we should use the following effective dielectric constant:

$$
\varepsilon \equiv \varepsilon_{12}=\frac{\left(\varepsilon_{\mathrm{I}}+2 \varepsilon_{M}\right)\left(2 \varepsilon_{\mathrm{II}}+3 \varepsilon_{M}\right)}{15 \varepsilon_{M}} .
$$

In the Appendix, we discuss the arguments for this model of dielectric screening in more detail and give explicit expressions for the effective dielectric constants $\varepsilon$ for different types of interaction. We also show that when the values of the dielectric constants of the QDs and the matrix are close enough, the screening of dipole-dipole and dipole-multipole interactions between two spherical QDs can be approximated by the same dielectric constant, as it has been done in our previous work. ${ }^{6,11}$

To simplify the expression for the matrix element of the interaction potential, we start from the Fourier expansion

$$
\frac{1}{\left|\mathbf{r}+\mathbf{r}_{I}-\mathbf{r}_{I I}\right|}=4 \pi \int \frac{d^{3} q}{(2 \pi)^{3}} \frac{e^{i \vec{q}\left(\mathbf{r}+\mathbf{r}_{I}-\mathbf{r}_{I I}\right)}}{q^{2}}
$$

which allows us to rewrite the matrix element in the following form:

$$
M \equiv\langle 2,4|V| 1,3\rangle=\frac{e^{2}}{2 \pi^{2} \varepsilon} \int d^{3} q e^{i \mathbf{q r}} S_{21}^{\mathrm{I}}(\mathbf{q}) S_{43}^{\mathrm{II}}(-\mathbf{q}),
$$

where

$$
S_{i j}^{\alpha}(\mathbf{q})=\int d^{3} r_{\alpha} e^{i \mathbf{q} \mathbf{r}_{\alpha}} \Psi_{i}^{*}\left(\mathbf{r}_{\alpha}\right) \Psi_{j}\left(\mathbf{r}_{\alpha}\right), \quad \alpha=\mathrm{I}, \mathrm{II} .
$$

In a two-band effective mass approximation, ${ }^{19}$ the wave function of strongly confined electrons and holes in a spherical QD with infinitely high potential walls has the following form: ${ }^{20}$

$$
\Psi_{k}\left(\mathbf{r}_{\alpha}\right) \equiv \Psi_{\kappa, i}\left(\mathbf{r}_{\alpha}\right)=u_{\kappa}\left(\mathbf{r}_{\alpha}\right) \psi_{i}\left(r_{\alpha}\right),
$$

where $u_{\kappa}$ is the Bloch amplitude $(\kappa=c, v)$,

$$
\psi_{i}\left(\mathbf{r}_{\alpha}\right)=R_{n_{i} l_{i}}\left(r_{\alpha}\right) Y_{l_{i} m_{i}}\left(\theta_{\alpha}, \phi_{\alpha}\right),
$$

is the envelope wave function,

$$
R_{n_{i} l_{i}}\left(r_{\alpha}\right)=\sqrt{\frac{2}{R_{\alpha}^{3}}} \frac{j_{l_{i}}\left(\xi_{n_{i} l_{i}} r_{\alpha} / R_{\alpha}\right)}{j_{l_{i}+1}\left(\xi_{n_{i} l_{i}}\right)}
$$

is the radial part, $Y_{l_{i} m_{i}}\left(\theta_{\alpha}, \phi_{\alpha}\right)$ is a spherical harmonic, $j_{l_{i}}$ is a spherical Bessel function, $\xi_{n_{i} l_{i}}$ is the $n_{i}$ th root of equation $j_{l_{i}}(x)=0$, and $R_{\alpha}$ is the radius of the QD. It should be noted that the important part of an intradot Coulomb interaction is taken into account by the effective mass approach.

Let us express $\mathbf{r}_{\alpha}$ as the sum of the radius vectors of an elementary cell $\mathbf{r}_{k_{\alpha}}$ and of the electron inside the cell $\mathbf{r}_{\alpha}^{\prime}$. By using the following properties of the envelope and Bloch functions:

$$
\psi_{i}\left(\mathbf{r}_{k_{\alpha}}+\mathbf{r}_{\alpha}^{\prime}\right) \approx \psi_{i}\left(\mathbf{r}_{k_{\alpha}}\right), \quad u_{\kappa_{1}}\left(\mathbf{r}_{k_{\alpha}}+\mathbf{r}_{\alpha}^{\prime}\right)=u_{\kappa_{1}}\left(\mathbf{r}_{\alpha}^{\prime}\right),
$$

we can replace the integral over the volume, $V_{\alpha}$, of the quantum dot, by the sum of the integrals over the volumes $\Omega$ of elementary cells. This allows us to represent $S_{i j}^{\alpha}$ as the products of the integrals over coordinates in the envelope space and the Bloch space

$$
S_{i j}^{\alpha}=s_{i j}^{\alpha} U_{i j}^{\alpha},
$$

where

$$
\begin{aligned}
& s_{i j}^{\alpha}=\Omega \sum_{k_{\alpha}} e^{i \mathbf{q} \mathbf{r}_{k_{\alpha}} \psi_{i}^{*}\left(\mathbf{r}_{k_{\alpha}}\right) \psi_{j}\left(\mathbf{r}_{k_{\alpha}}\right),}
\end{aligned}
$$

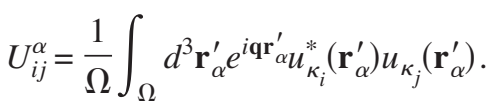

After the replacement of the sum over the elementary cells with the integral over the volume of the nanocrystal, we obtain

$$
s_{i j}^{\alpha}=\int d^{3} r_{\alpha} e^{i \mathbf{q} \mathbf{r}_{\alpha}} \psi_{i}^{*}\left(\mathbf{r}_{\alpha}\right) \psi_{j}\left(\mathbf{r}_{\alpha}\right) .
$$

The expansion of the plane wave $e^{i \mathbf{q r} \mathbf{r}_{\alpha}}$ in terms of spherical waves, expressing the integral of product of three spherical functions through Clebsch-Gordan coefficients ${ }^{21}$ and noting the spherical symmetry of quantum dots, allows us to transform the integration over the angular coordinates of vector $\mathbf{r}_{\alpha}$ in Eq. (11) into the following sum:

$$
\begin{aligned}
s_{i j}^{\alpha}( \pm \mathbf{q})= & \sqrt{\frac{2 l_{j}+1}{2 l_{i}+1}} \sum_{l_{\alpha}=0}^{l_{i}+l_{j}}( \pm i)^{l}(2 l+1) C_{l_{j} 0, l_{\alpha} 0}^{l_{i} 0} C_{l_{j} m_{j}, l_{\alpha} 0}^{l_{i} m_{i}} \\
& \times \int_{0}^{R_{\alpha}} d r_{\alpha} r^{2} j_{l_{\alpha}}\left(q r_{\alpha}\right) R_{n_{i} l_{i}}^{*}\left(r_{\alpha}\right) R_{n_{j} l_{j}}\left(r_{\alpha}\right),
\end{aligned}
$$

where $C_{l_{j} m_{j} l_{\alpha} l^{0}}^{l_{i}}$ is the Clebsch-Gordan coefficient.

In the long-wave approximation, $q a \ll 1$, where $a$ is the lattice constant of the semiconductor, we can decompose $e^{i \mathbf{q r}_{\alpha}^{\prime}}$ into a Taylor series and estimate the integrals $U_{i j}^{\alpha}$ of the Bloch amplitudes. When the functions of the initial and final state belong to the same energy band, the first nonzero term of $U_{i j}^{\alpha}$ is 1 . Then we have

$$
U_{i j}^{\alpha}\left(\kappa_{i}=\kappa_{j}=\kappa\right) \approx \frac{1}{\Omega} \int_{\Omega} d^{3} r_{\alpha}^{\prime}\left|u_{\kappa}\left(\mathbf{r}_{\alpha}^{\prime}\right)\right|^{2}=1 .
$$

Otherwise, for interband transitions, we obtain

$$
U_{i j}^{\alpha}\left(\kappa_{i} \neq \kappa_{j}\right) \approx \frac{i \mathbf{q}}{\Omega} \int_{\Omega} d^{3} r_{\alpha}^{\prime} u_{\kappa_{i}}^{*}\left(\mathbf{r}_{\alpha}^{\prime}\right) \mathbf{r}_{\alpha}^{\prime} u_{\kappa_{j}}\left(\mathbf{r}_{\alpha}^{\prime}\right)=i \mathbf{q} \mathbf{r}_{\kappa_{i}, \kappa_{j}}^{\alpha} .
$$

The matrix element of the radius-vector inside the elementary cell between Bloch functions $\mathbf{r}_{\kappa_{2}, \kappa_{1}}^{\alpha}$ can be expressed via the Kane parameter $P_{\alpha}=\hbar^{2} / m_{0}\left\langle S_{\alpha}|\partial / \partial z| Z_{\alpha}\right\rangle=\hbar \sqrt{E_{p, \alpha} /\left(2 m_{0}\right)}$ and the energy gap $E_{g, \alpha}$ as follows:

$$
\left|\mathbf{r}_{c v}^{\alpha}\right|=P_{\alpha} / E_{g, \alpha},
$$

where $E_{p, \alpha}$ is the Kane energy. 
Using Eqs. (13) and (14), we obtain the following expression for matrix element (8):

$$
M_{\ell}^{\alpha}=\frac{e^{2}}{2 \pi^{2} \varepsilon} \int \frac{d^{3} q}{q^{2}} e^{i \mathbf{q r}} \xi_{\ell}^{\alpha} S_{21}^{\mathrm{I}}(\mathbf{q}) s_{43}^{\mathrm{II}}(-\mathbf{q}), \quad \ell=0,1,2 .
$$

Here, we introduce the index $\ell$ that determines the number of interband transitions involved in scattering, the upper index $\alpha$ in $M_{\ell}^{\alpha}$ denotes the QD (I or II) number where the interband interaction occurs, $\xi_{\ell}^{\alpha}$ is the function that determines the mutual orientation of $\mathbf{q}$ and $\mathbf{r}_{c v}^{\alpha}$,

$$
\xi_{0}^{\alpha}=1, \quad \xi_{1}^{\alpha}= \pm i \mathbf{q} \mathbf{r}_{c v}^{\alpha}, \quad \xi_{2}^{\alpha}=\left(\mathbf{q} \mathbf{r}_{c v}^{\mathrm{I}}\right)\left(\mathbf{q} \mathbf{r}_{v c}^{\mathrm{II}}\right),
$$

where $e \mathbf{r}_{c v}^{\alpha}$ are the dipole moments of the interband transitions. In this and other similar expressions for interbandintraband transitions $(\ell=1)$, the plus sign is used when the interband transition occurs in the first quantum dot $(\alpha=\mathrm{I})$ and a minus sign for the opposite case $(\alpha=$ II). Integration over the angles between $\mathbf{q}$ and $\mathbf{r}$ and change in the integration order of $r$ and $q$ allow us to evaluate the integral over $q$ analytically. We obtain the following expression:

$$
\begin{aligned}
& M_{\ell}^{\alpha}=\frac{e^{2}}{\varepsilon} \frac{X_{\ell}^{\alpha}}{r^{\ell+1}}, \\
& X_{0}^{\alpha}=I_{0}, \quad X_{1}^{\alpha}= \pm I_{1} \mathbf{n}_{r} \mathbf{r}_{c v}^{\alpha}, \\
& X_{2}^{\alpha}=I_{1} \mathbf{r}_{c v}^{\mathrm{I}} \mathbf{r}_{c v}^{\mathrm{II}}-I_{2}\left(\mathbf{n}_{r} \mathbf{r}_{c v}^{\mathrm{I}}\right)\left(\mathbf{n}_{r} \mathbf{r}_{c v}^{\mathrm{II}}\right) .
\end{aligned}
$$

Here $\mathbf{n}_{r}=\mathbf{r} / r$,

$$
\begin{aligned}
I_{\ell}= & \frac{2}{\pi} \sum_{l_{\mathrm{I}}=0}^{l_{1}+l_{2}} \sum_{l_{\mathrm{II}}=0}^{l_{3}+l_{4}} \mathfrak{C}_{\mathrm{I}}^{+} \mathfrak{C}_{\mathrm{II}}^{-} \\
& \times \int_{0}^{R_{\mathrm{I}}} \int_{0}^{R_{\mathrm{II}}} d r_{\mathrm{I}} d r_{\mathrm{II}} r_{\mathrm{I}}^{2} r_{\mathrm{II}}^{2} \Re_{\mathrm{I}} \Re_{\mathrm{II}} Q_{l_{\mathrm{I}}, l_{\mathrm{II}}}^{\ell}, \quad \ell=0,1,2
\end{aligned}
$$

are the multipole amplitudes that come from the integration over the coordinates of the envelope wave functions,

$$
\begin{aligned}
& \mathfrak{C}_{\alpha}^{ \pm}=( \pm i)^{l_{\alpha}}\left(2 l_{\alpha}+1\right) \sqrt{\frac{2 l_{j}+1}{2 l_{i}+1}} C_{l_{j} 0, l_{\alpha} 0}^{l_{i} 0} C_{l_{j} m_{j}, l_{\alpha} 0}^{l_{i} m_{i}}, \\
& \Re_{\alpha}=R_{\mathbf{n}_{\alpha}^{\prime} \prime^{\prime}}^{*}\left(r_{\alpha}\right) R_{n_{\alpha} l_{\alpha}^{l}}\left(r_{\alpha}\right), \\
& Q_{l_{\mathrm{I}}, l_{\mathrm{II}}}^{\ell}=r^{\ell+1} \int_{0}^{\infty} d q q^{\ell} j_{\ell}(q r) j_{l_{\mathrm{I}}}\left(q r_{\mathrm{I}}\right) j_{l_{\mathrm{II}}}\left(q r_{\mathrm{II}}\right) \\
& =2^{\ell-3} \pi^{3 / 2}\left(\frac{r_{\mathrm{I}}}{r}\right)^{l_{\mathrm{I}}}\left(\frac{r_{\mathrm{II}}}{r}\right)^{l_{\mathrm{II}}} \\
& \times \Gamma\left[\begin{array}{c}
\left(2 \ell+l_{\mathrm{I}}+l_{\mathrm{II}}+1\right) / 2 \\
l_{\mathrm{I}}+3 / 2, l_{\mathrm{II}}+3 / 2,1-\left(l_{\mathrm{I}}+l_{\mathrm{II}}\right) / 2
\end{array}\right] \\
& \times F_{4}\left(\frac{l_{\mathrm{I}}+l_{\mathrm{II}}}{2}, \frac{2 \ell+l_{\mathrm{I}}+l_{\mathrm{II}}+1}{2} ; l_{\mathrm{I}}+\frac{3}{2}, l_{\mathrm{II}}\right. \\
& \left.+\frac{3}{2} ; \frac{r_{1}^{2}}{r^{2}} ; \frac{r_{\text {II }}^{2}}{r^{2}}\right) \text {, }
\end{aligned}
$$

where $F_{4}\left(a, b ; c, c^{\prime} ; x ; y\right)$ is the Appel's fourth hypergeometric function, ${ }^{22}$

$$
F_{4}\left(a, b ; c, c^{\prime} ; x ; y\right)=\sum_{k, l=0}^{\infty} \frac{(a)_{k+l}(b)_{k+l}}{(c)_{k}\left(c^{\prime}\right)_{l}} \frac{x^{k} y^{l}}{k ! l !},
$$

$(a)_{k}=\Gamma(a+k) / \Gamma(a)$ is a Pochhammer symbol.

Next, we introduce orientational factors that are independent of the values of the transition dipole moments,

$$
\begin{aligned}
\chi_{0}= & I_{0}, \quad \chi_{1}^{\alpha}= \pm I_{1} \cos \theta_{\alpha}, \quad \chi_{2}=I_{1} \sin \theta_{\mathrm{I}} \sin \theta_{\mathrm{II}} \cos \phi \\
& +\left(I_{1}-I_{2}\right) \cos \theta_{\mathrm{II}} \cos \theta_{\mathrm{II}} ;
\end{aligned}
$$

the matrix elements can be rewritten in the following way:

$$
M_{0}=\frac{e^{2}}{\varepsilon} \frac{\chi_{0}}{r}, \quad M_{1}^{\alpha}=\frac{e^{2}}{\varepsilon} \frac{\chi_{1}^{\alpha}}{r^{2}} r_{c v}^{\alpha}, \quad M_{2}=\frac{e^{2}}{\varepsilon} \frac{\chi_{2}}{r^{3}} r_{c v}^{\mathrm{I}} r_{c v}^{\mathrm{II}} .
$$

Hereafter, we omit the superscripts $\alpha$ in the expressions for intraband-intraband $(\ell=0)$ and interband-interband $(\ell=2)$ transitions.

It should be noted that the infinitely high potential walls approximation significantly reduces the probability of transition between the states with different principal quantum numbers $n_{i}$, so in this paper, our consideration is restricted to transitions between the states, which differ only by the angular moment values. This limitation stems from the orthogonality of the radial wave functions (10) by $n_{i}$ and thus from the elimination of the terms proportional to $\left(r_{\alpha} / r\right)^{2 k}, k$ $=0, \infty$ [see Eqs. (21) and (22)]. The transitions between the states with different principal quantum numbers can be considered more precisely in the approximation of finite potential walls by replacing the radial parts of the envelope wave functions and extending the integration limits in Eq. (18), as discussed in our previous work. ${ }^{6}$ In order to maintain the spherical symmetry of the task, one must neglect the presence of the wave function of the first QD in the second QD and vice versa. For example, the integration over $r_{\mathrm{I}}$ should be performed inside the QDs $\left(0<r_{\mathrm{I}}<R_{\mathrm{I}}\right)$ and inside the spherical layer of matrix $\left(R_{\mathrm{I}}<r_{\mathrm{I}}<r-R_{\mathrm{II}}\right)$.

When the quantum numbers of the initial and final states are the same $\left(\nu_{1}=\nu_{2}, \nu_{3}=\nu_{4}, \nu_{i}=\left\{n_{i}, l_{i}, m_{i}\right\}\right)$, we have a dipole-allowed transition, and the function $I_{\ell}$ is reduced to

$$
I_{\ell, \mathrm{a}}=(2 \ell-1) ! !,
$$

where $(2 \ell-1) ! !=1 \cdot 3 \cdot 5 \cdot \ldots \cdot(2 \ell-1)$ is a double factorial function. For instance, Eq. (24) gives $I_{0, \mathrm{a}}=1, I_{1, \mathrm{a}}=1$, and $I_{2, \mathrm{a}}=3$, and the matrix element for dipole-allowed, interbandinterband transitions takes the well-known form ${ }^{4,12}$

$$
M_{2, \mathrm{a}}=\frac{e^{2}}{\varepsilon r^{3}}\left[\mathbf{r}_{c v}^{\mathrm{I}} \mathbf{r}_{c v}^{\mathrm{II}}-\left(3 \mathbf{n}_{r} \mathbf{r}_{c v}^{\mathrm{I}}\right)\left(\mathbf{n}_{r} \mathbf{r}_{c v}^{\mathrm{II}}\right)\right] .
$$

Let us consider the selection rules defined by expressions (17) and (18). Condition

$$
C_{l_{j} 0, l_{\alpha} 0}^{l_{i} 0} C_{l_{j} m_{j}, l_{\alpha} 0}^{l_{i} m_{i}} \neq 0
$$

implies the following selection rules for the transitions due to Coulomb interaction:

$$
\left|l_{j}-l_{\alpha}\right| \leq l_{i} \leq\left|l_{j}+l_{\alpha}\right|,
$$

$l_{i}+l_{j}+l_{\alpha}=$ even number, 


$$
m_{i}=m_{j}
$$

Additional selection rules arise from the condition $\Gamma\left(1-\left(l_{\mathrm{I}}\right.\right.$ $\left.\left.+l_{\mathrm{II}}\right) / 2\right) \neq \infty \quad$ [see Eq. (21)], which implies $\left(l_{\mathrm{I}}+l_{\mathrm{II}}\right) / 2$ $=\mathrm{a}$ half-integer number. This condition will be satisfied only when $l_{\mathrm{I}}$ and $l_{\mathrm{II}}$ have opposite parities. Since Eq. (26) should also be satisfied, we conclude that the matrix element is nonzero when the sums of the angular moments of the initial and final states have opposite parities, i.e., in two cases: (1) $l_{1}+l_{2}=$ an odd number and $l_{3}+l_{4}$ $=$ an even number and (2) $l_{1}+l_{2}=$ an even number and $l_{3}$ $+l_{4}=$ an odd number.

It is useful to derive expressions for the squared modulus of matrix elements averaged by directions of transition dipole moments and their maximal values,

$$
\begin{aligned}
\overline{\left|M_{0}\right|^{2}}= & \max \left|M_{0}\right|^{2}=\frac{e^{4}\left|I_{0}\right|^{2}}{\varepsilon^{2} r^{2}}, \\
\overline{\left|M_{1}^{\alpha}\right|^{2}}= & \frac{1}{3} \frac{e^{4}}{\varepsilon^{2}} \frac{\left|I_{1}\right|^{2}}{r^{4}}\left|\mathbf{r}_{c v}^{\alpha}\right|^{2}, \quad \max \left|M_{1}^{\alpha}\right|^{2}=\frac{e^{4}}{\varepsilon^{2}} \frac{\left|I_{1}\right|^{2}}{r^{4}}\left|\mathbf{r}_{c v}^{\alpha}\right|^{2}, \\
\overline{\left|M_{2}\right|^{2}}= & \frac{1}{3} \frac{e^{4}}{\varepsilon^{2} r^{6}}\left|\mathbf{r}_{c v}^{\mathrm{I}}\right|^{2}\left|\mathbf{r}_{c v} \mathrm{II}\right|^{2}\left\{\left|I_{1}\right|^{2}-\frac{1}{3}\left(I_{1}^{*} I_{2}+I_{1} I_{2}^{*}\right)+\frac{1}{3}\left|I_{2}\right|^{2}\right\}, \\
& \max \left|M_{2}\right|^{2}=\frac{e^{4}}{\varepsilon^{2} r^{6}}\left|\mathbf{r}_{c v}^{\mathrm{I}}\right|^{2}\left|\mathbf{r}_{c v}^{\mathrm{II}}\right|^{2}\left|I_{1}-I_{2}\right|^{2} .
\end{aligned}
$$

Conditions for $\left|M_{\ell}\right|^{2}$ maximality over the angular variables are determined by the orientation factors $\chi_{\ell}$ [see Eq. (23)]. Intraband-intraband transitions $(\ell=0)$ do not depend on lattice orientation because they do not include any coordinates of Bloch space and because QDs are spherically symmetric. For $\ell=1$, there are two conditions: (1) $\theta_{\alpha}=0$ and (2) $\theta_{\alpha}=\pi$, i.e., $\left|M_{1}\right|^{2}$ is maximal when the transition dipole moment is parallel to the vector $\mathbf{r}$ connecting the centers of the QDs. For $\ell=2$, we have four orientations of the transition dipole moments, which maximize the matrix element: (1) $\theta_{\mathrm{I}}=0$, $\theta_{\mathrm{II}}=0$; (2) $\theta_{\mathrm{I}}=0, \theta_{\mathrm{II}}=\pi ;$; (3) $\theta_{\mathrm{I}}=\pi, \theta_{\mathrm{II}}=0$; and (4) $\theta_{\mathrm{I}}=\pi, \theta_{\mathrm{II}}$ $=\pi$, i.e., both vectors $\mathbf{r}_{\mathrm{I}}$ and $\mathbf{r}_{\mathrm{II}}$ should be parallel to $\mathbf{r}$.

It is convenient to designate the real transitions in a double QD by a notation commonly used in the theory of Auger processes in bulk semiconductors and heterostructures $^{23,24}$ (CVVC, CVCC, CCCC,...). The letters $\mathrm{C}$ and $\mathrm{V}$ denote the conduction and the valence bands, respectively, but in this context the first two letters describe the transition in the first QD and the last two in the second QD. The matrix elements (17), with respective values for the index $\ell$, describe the probability amplitudes of the processes listed in Table I.

The expressions for the matrix elements obtained in this section allow the determination of important parameters that characterize the resonant interaction of two QDs. For example, the rate of resonant energy transfer between two nondegenerated states is given by the expression

$$
\gamma=\frac{2}{\hbar^{2}}\left|M_{\ell}\right|^{2} \frac{\Gamma}{\Gamma^{2}+\Delta^{2}}=\frac{\Omega_{\ell}^{2}}{2} \frac{\Gamma}{\Gamma^{2}+\Delta^{2}},
$$

where $\Gamma$ is the transition dephasing rate and $\Delta$ is the detuning between the transition frequencies of two interacting sys-
TABLE I. Correspondence between the matrix elements (17) and the transitions in a double QD.

\begin{tabular}{c}
\hline \hline Intraband-intraband transitions \\
$M_{0}:$ CCCC, CCVV, VVCC, VVVV \\
\hline Interband-intraband transitions \\
$M_{1}^{\mathrm{I}}:$ VCCC, CVCC, CVVV, VCVV \\
$M_{1}^{\mathrm{II}}:$ CCVC, CCCV, VVCV, VVVC \\
\hline Interband-interband transitions \\
$M_{2}:$ CVVC, VCCV \\
\hline \hline
\end{tabular}

tems. The parameter $\Omega_{\ell}=2\left|M_{\ell}\right| / \hbar$, following from a density matrix description of interacting two-level systems, ${ }^{12}$ plays an important role in the theory of resonant energy transfer. This is the frequency characterizing the oscillations of the excited states population in resonantly interacting two-level systems. The relationship between $\Omega_{\ell}$, the population relaxation rate $\gamma_{\text {II }}$ of the final state, and the transition dephasing rate $\Gamma$ determines the regime of resonant coupling: incoherent irreversible $\left(\Omega_{\ell} \ll \gamma_{\text {II }}, \Gamma\right)$, incoherent reversible $\left(\gamma_{\text {II }}<\Omega_{\ell}\right.$ $<\Gamma)$, or coherent $\left(\Omega_{\ell} \gg \gamma_{\mathrm{II}}, \Gamma\right)$. Notice that Eq. (30) based on the Fermi's Golden Rule is valid for the description of the energy transfer rates in the following cases: $\Omega_{\ell} \ll \gamma_{\text {II }}, \Gamma$ and $\gamma_{\mathrm{II}}<\Omega_{\ell}<\Gamma$.

In the next section, we perform numerical calculations to determine $\max \Omega_{\ell}$, assuming that the mutual orientation of the dipole moments of the interband transitions satisfies one of the conditions for maximality of $M_{\ell}$.

\section{NUMERICAL RESULTS}

Let us consider the transition rate dependencies on geometric parameters for double QDs of two different materials: $\mathrm{CdSe}$ and $\mathrm{InSb}$. The relevant material parameters are listed in Table II. Hereafter, we assume that the matrix material is $\mathrm{SiO}_{2}$, so the dielectric constant at typical transition frequencies is $\varepsilon_{M}=2.13$ (Ref. 28).

We perform the calculations for materials with different band gaps because it is expected that the energy gap value will significantly affect the relative magnitudes of the matrix elements for different types of transitions. Among the intraband-intraband processes mentioned in Table I, only CCCC and VVVV are of interest for certain QD materials. The effective masses of electrons and holes in CdSe and InSb differ significantly, $m_{v} / m_{c} \sim 10$, so the energy resonance conditions for CCVV and VVCC processes lead to unrealistic values for quantum dot radii for the lowest allowed transitions. Due to the similarity of the expressions for the energy spectrum in the conduction and valence bands in

TABLE II. Material parameters of CdSe (Refs. 25 and 26) and InSb (Ref. 27). $m_{h h}=m_{v}$ is the heavy hole effective mass.

\begin{tabular}{llcccc}
\hline \hline & & & $E_{p}$ & $E_{g}$ & \\
& $m_{c}\left(m_{0}\right)$ & $m_{h h}\left(m_{0}\right)$ & $(\mathrm{eV})$ & $(\mathrm{eV})$ & $\varepsilon_{\alpha}$ \\
\hline $\mathrm{CdSe}$ & 0.11 & 1.14 & 17.5 & 1.76 & 5.8 \\
$\mathrm{InSb}$ & 0.0139 & 0.44 & 23.42 & 0.17 & 16 \\
\hline \hline
\end{tabular}



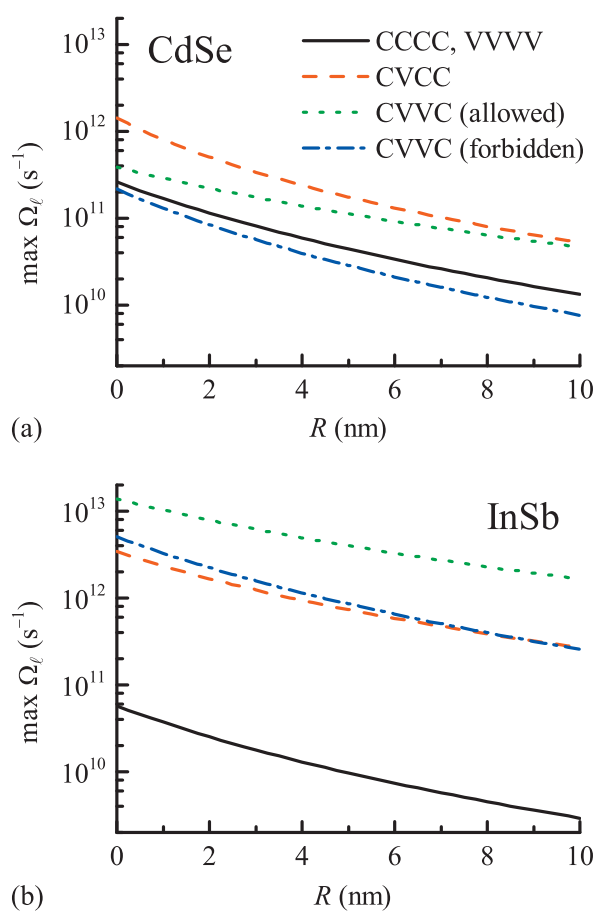

FIG. 2. Parameter $\max \Omega_{\ell}$ as a function of the intersurface distance for different types of electronic transitions in a double QD: CCCC, VVVV, CVCC, and CVVC. We consider that the first QD radius has a fixed value $R_{\mathrm{I}}=4 \mathrm{~nm}$, and the second $\mathrm{QD}$ radius $R_{\mathrm{II}}$ always satisfies the resonance condition $\omega_{\mathrm{I}}\left(R_{\mathrm{I}}\right)=\omega_{\mathrm{II}}\left(R_{\mathrm{II}}\right)$, where $\omega_{\alpha}$ is the corresponding transition frequency. CCCC transition: $\{c 110 ; c 100\}_{\mathrm{I}},\{c 100 ; c 120\}_{\mathrm{II}}, R_{\mathrm{II}}=6 \mathrm{~nm}$; CVCC transition: $\{c 100 ; v 100\}_{\mathrm{I}}, \quad\{c 100 ; c 110\}_{\mathrm{II}}, \quad R_{\mathrm{II}}=3.85 \mathrm{~nm} ;$ dipole-allowed CVVC transition: $\{c 100 ; v 100\}_{\mathrm{I}},\{v 110 ; c 110\}_{\mathrm{II}}, R_{\mathrm{II}}=5.72 \mathrm{~nm}$; and dipoleforbidden CVVC transition: $\{c 100 ; v 100\}_{\mathrm{I}},\{v 110 ; c 100\}_{\mathrm{II}}, R_{\mathrm{II}}=5.68 \mathrm{~nm}$.

a two-band approximation, CCCC and VVVV processes will have the same values for the interaction matrix elements, and so they will be considered together.

Figures 2(a) and 2(b) show the dependencies of $\max \Omega_{\ell}$ on the distance between the surfaces of interacting QDs $(R$ $\left.=r-R_{\mathrm{I}}-R_{\mathrm{II}}\right)$ for the lowest allowed transitions. The dependency on $R$ will not be the same as that on center-to-center distance $r$, but it is more convenient for comparison of the calculated results for QD pairs with different radii. For CdSe QDs, we see that the dominant process is CVCC. Thus, the interband-intraband processes can be considered as another effective mechanism of resonant energy transfer as well as interband-interband processes. For InSb, the frequencies $\Omega_{\ell}$ for CVVC and CVCC have much higher values than in CdSe since InSb has higher values of transition dipole moments than CdSe. At the same time, the rates of intraband-intraband processes in InSb QDs are lower than in CdSe.

It should be noted that resonant electrostatic interaction of charge carriers inside QDs cannot be considered as a relaxation mechanism itself since the total energy of the interacting particles is conserved, and so these transitions are fully reversible in the absence of the bath. Transitions between single-particle states due to electrostatic correlations play an important role as relaxation mediators because they can bring the system into the states with a much faster relaxation rate than the initial state.

Let us consider the dependency of $\max \Omega_{\ell}$ on the radius of the first QD at the fixed intersurface distance depicted in
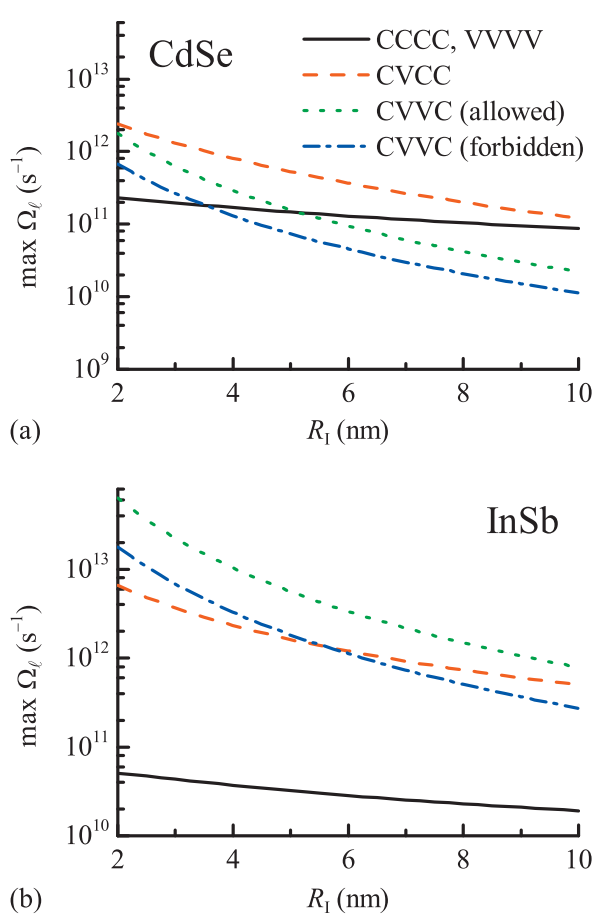

FIG. 3. Parameter $\max \Omega_{\ell}$ as function of the first QD radius $R_{\mathrm{I}}$ at intersurface distance of $R=1 \mathrm{~nm}$. As before, $R_{\mathrm{II}}$ is calculated from the resonance condition; transitions are the same as in Fig. 2 .

Fig. 3. This figure shows that the $\max \Omega_{\ell}$ for intrabandintraband transitions decreases much more slowly than that for other processes with increasing $R_{\mathrm{I}}$. We also observe the domination of dipole-allowed CVVC process in InSb QDs, but CVCC process is also effective and overcomes dipoleforbidden CVVC process in QDs with $R_{\mathrm{I}} \lesssim 6 \mathrm{~nm}$.

\section{CONCLUSION}

In this paper, we have studied the probability amplitudes for electron-electron scattering processes in a double QD under strong confinement. We assume that the electrostatic interaction between the QDs is described by the Coulomb potential and dielectric screening is taken into account by the effective dielectric constant. We discuss the expressions for this effective dielectric constant and determine the boundaries of its possible variations if used as a fitting parameter. Numerical calculations have been performed for the dependencies of the matrix elements on interdot distance, QD size, and material parameters. It has been shown that in some cases, the interband-intraband and intraband-intraband transitions can have higher rates than the commonly considered interband-interband transitions.

\section{ACKNOWLEDGMENTS}

Three of the authors (S.Yu.K., A.V.F., and A.V.B.) are grateful to the RFBR (Grant Nos. 09-02-00333 and 09-0201439) and the Ministry of Education and Science of the Russian Federation (Grant Nos. 2.1.1/1933 and 2.1.1/1880) for partial financial support of this work. 


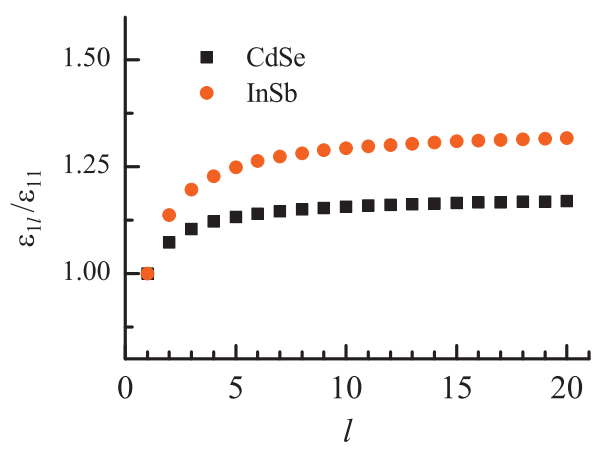

FIG. 4. Ratios of effective dielectric constants $\varepsilon_{1 l} / \varepsilon_{11}$ for the first 20 multipole terms for double QDs of CdSe and InSb. The dielectric constants for $\mathrm{CdSe}$ and $\mathrm{InSb}$ are listed in Table II; the matrix material is $\mathrm{SiO}_{2}\left(\varepsilon_{M}\right.$ $=2.13$ ).

\section{APPENDIX: DIELECTRIC SCREENING OF TWO ELECTROSTATICALLY COUPLED QDS}

The assumption that the interaction between two spherical QDs is described by the Coulomb potential with effective dielectric constant offers many advantages. In particular, it simplifies the consideration of higher multipoles and allows a straightforward generalization of many-body theory for ordinary solids ${ }^{14,29}$ to QD solids. The simplicity of the expressions for probability amplitudes of electron-electron scattering makes the ordered structures of spherical QDs a good model system for the investigation of various effects connected with electronic correlation, such as resonant energy transfer and carrier multiplication. ${ }^{30}$ However, the applicability of the effective dielectric constant derived for dipoledipole interactions when transitions in one of the quantum dots are not dipole-allowed, i.e., the envelope angular momenta of the initial and final states are different, is questionable.

The first reason for the usage of the same effective dielectric constant is that the charge distribution inside the QD remains spherically symmetric for all envelope states. So, according to classical electrostatics, ${ }^{31}$ the potential generated by two oppositely charged, spherically symmetric charge distributions can still be described by the point dipole located at the center of the sphere. Possible deviations from the point dipole approximation, arising from the asymmetry between the envelope wave functions of the electrons and holes, will be small if the transition occurs between states with the same principal quantum numbers.

A second reason is that the effective dielectric constant describing the screening of the interaction between the $l_{\mathrm{I}}$ th and $l_{\mathrm{II}}$ th multipoles in spherical QDs, ${ }^{4,17,18}$

$$
\varepsilon_{l_{\mathrm{I}} l_{\mathrm{II}}}\left(\varepsilon_{\mathrm{I}}, \varepsilon_{\mathrm{II}}, \varepsilon_{M}\right)=\frac{\varepsilon_{M}}{f_{l_{\mathrm{I}}}\left(\varepsilon_{\mathrm{I}} / \varepsilon_{M}\right) f_{l_{\mathrm{II}}}\left(\varepsilon_{\mathrm{II}} / \varepsilon_{M}\right)},
$$

varies slowly over the lowest multipole moments $l_{\mathrm{I}}$ and $l_{\mathrm{II}}$ for typical values of the dielectric constants of semiconductors and glasses. This is clearly seen in Fig. 4, which depicts the ratio of effective dielectric constants for dipole-multipole and dipole-dipole interactions $\varepsilon_{1 l} / \varepsilon_{11}(l=1, \ldots, 20)$. Note that the closer the values of $\varepsilon_{\alpha}$ and $\varepsilon_{M}$, the slower the value of $\varepsilon_{1 l}$ will vary over $l$. The upper limit of $\varepsilon_{1 l} / \varepsilon_{11}$ is given by the expression

$$
\lim _{l \rightarrow \infty} \frac{\varepsilon_{1 l}}{\varepsilon_{11}}=\frac{3\left(\varepsilon_{\mathrm{II}}+\varepsilon_{M}\right)}{2 \varepsilon_{\mathrm{II}}+2 \varepsilon_{M}},
$$

so we have $\varepsilon_{1 \infty} / \varepsilon_{11} \approx 1.18$ for a double QD made from CdSe and $\varepsilon_{1 \infty} / \varepsilon_{11} \approx 1.34$ for a double QD made from InSb. Thus, in order to approximately consider the static screening of interactions in spherical QDs formed from direct-band semiconductor, it is sufficient to assume that $f_{l_{\alpha}}$ for any $l_{\alpha}$ is equal to the field factor of the first leading term, e.g., $f_{l_{\alpha}} \approx f_{1}$ for dipole-allowed transition and $f_{l_{\alpha}} \approx f_{2}$ for dipole-forbidden transition.

If both transitions are dipole-allowed, the leading term in the multipole expansion of the potential (3) corresponds to dipole-dipole interaction. Thus, the effective constant is determined by the expression

$$
\varepsilon_{11}=\frac{\left(\varepsilon_{\mathrm{I}}+2 \varepsilon_{M}\right)\left(\varepsilon_{\mathrm{II}}+2 \varepsilon_{M}\right)}{9 \varepsilon_{M}} .
$$

This situation is realized for interband-interband transitions. If one of the transitions is dipole-forbidden (i.e., angular moments of initial and final states are different), the leading term will correspond to dipole-quadrupole interaction, so one should use the following effective dielectric constant:

$$
\varepsilon_{12}=\frac{\left(\varepsilon_{\mathrm{I}}+2 \varepsilon_{M}\right)\left(2 \varepsilon_{\mathrm{II}}+3 \varepsilon_{M}\right)}{15 \varepsilon_{M}} .
$$

Finally, if both transitions are dipole-forbidden, we have

$$
\varepsilon_{22}=\frac{\left(2 \varepsilon_{I}+3 \varepsilon_{M}\right)\left(2 \varepsilon_{I I}+3 \varepsilon_{M}\right)}{25 \varepsilon_{M}} .
$$

The effective dielectric constant for different multipole terms varies between $\varepsilon_{12}$ and $\varepsilon_{1 \infty}=\left(\varepsilon_{\mathrm{I}}+2 \varepsilon_{M}\right)\left(\varepsilon_{\mathrm{II}}+\varepsilon_{M}\right) / 6 \varepsilon_{M}$, so when $\varepsilon_{\alpha} \gg \varepsilon_{M}$, one may treat $\varepsilon$ as an adjustable parameter to achieve a better agreement between calculations and experimental results for dipole-multipole interactions.

Similarly, in case of real intraband-intraband transitions, the leading term will correspond to quadrupole-quadrupole interaction, so the effective screening constant varies between $\varepsilon_{22}$ and

$$
\varepsilon_{2 \infty}=\frac{\left(2 \varepsilon_{\mathrm{I}}+3 \varepsilon_{M}\right)\left(\varepsilon_{\mathrm{II}}+\varepsilon_{M}\right)}{10 \varepsilon_{M}} .
$$

${ }^{1}$ S. V. Gaponenko, Optical Properties of Semiconductor Nanocrystals (Cambridge University Press, Cambridge, 1998).

${ }^{2}$ G. Allan and C. Delerue, Phys. Rev. B 75, 195311 (2007).

${ }^{3}$ D. L. Andrews and J. M. Leeder, J. Chem. Phys. 130, 184504 (2009).

${ }^{4}$ R. Baer and E. Rabani, J. Chem. Phys. 128, 184710 (2008).

${ }^{5}$ C. Curutchet, A. Franceschetti, A. Zunger, and G. D. Scholes, J. Phys. Chem. C 112, 13336 (2008).

${ }^{6}$ S. Yu. Kruchinin, A. V. Fedorov, A. V. Baranov, T. S. Perova, and K. Berwick, Phys. Rev. B 78, 125311 (2008).

${ }^{7}$ C. R. Kagan, C. B. Murray, and M. G. Bawendi, Phys. Rev. B 54, 8633 (1996).

${ }^{8}$ L. Xu, J. Xu, Z. Ma, W. Li, X. Huang, and K. Chen, Appl. Phys. Lett. 89, 033121 (2006).

${ }^{9}$ K. Nishibayashi, T. Kawazoe, M. Ohtsu, K. Akahane, and N. Yamamoto, Appl. Phys. Lett. 93, 042101 (2008).

${ }^{10}$ G. D. Scholes and D. L. Andrews, Phys. Rev. B 72, 125331 (2005). 
${ }^{11}$ S. Yu. Kruchinin, A. V. Fedorov, A. V. Baranov, T. S. Perova, and K Berwick, Phys. Rev. B 81, 245303 (2010).

${ }^{12}$ V. M. Agranovich and M. D. Galanin, Electronic Excitation Energy Transfer in Condensed Matter (North-Holland, Amsterdam, 1982).

${ }^{13}$ A. Nazir, B. W. Lovett, S. D. Barrett, J. H. Reina, and G. A. D. Briggs, Phys. Rev. B 71, 045334 (2005).

${ }^{14}$ A. L. Fetter and J. D. Walecka, Quantum Theory of Many-Particle Systems (McGraw-Hill, New York, 1971).

${ }^{15}$ H. Haken, Quantum Field Theory of Solids: An Introduction (NorthHolland, Amsterdam, 1983).

${ }^{16}$ A. Franceschetti and A. Zunger, Phys. Rev. Lett. 78, 915 (1997).

${ }^{17}$ C. J. F. Böttcher, Theory of Electric Polarization, 2nd ed. (Elsevier, Amsterdam, 1973), Vol. 1.

${ }^{18}$ L. Bányai and S. W. Koch, Semiconductor Quantum Dots (World Scientific, Singapore, 1993).

${ }^{19}$ The effective mass approximation is commonly used approach for analytical calculations of the eigenfunctions and eigenvalues of semiconductor electronic subsystem including semiconductor nanostructures. See, for example, E. L. Ivchenko and G. E. Pikus, Superlattices and Other Het- erostructures (Springer-Verlag, Berlin, 1997).

${ }^{20}$ Al. L. Efros and A. L. Efros, Sov. Phys. Semicond. 16, 772 (1982).

${ }^{21}$ Quantum Theory of Angular Momentum, edited by D. A. Varshalovich, A. N. Moskalev, and V. K. Khersonskii (World Scientific, Singapore, 1988)

${ }^{22}$ A. P. Prudnikov, Y. A. Brychkov, and O. I. Marichev, Special Functions, Integrals and Series, Vol. 2 (Gordon and Breach, New York, 1990).

${ }^{23}$ M. Takeshima, Phys. Rev. B 26, 917 (1982).

${ }^{24}$ V. N. Abakumov, V. I. Perel, and I. N. Yassievich, Nonradiative Recombination in Semiconductors (North-Holland, Amsterdam, 1991).

${ }^{25}$ S. Ninomiya and S. Adachi, J. Appl. Phys. 78, 4681 (1995).

${ }^{26}$ D. J. Norris and M. G. Bawendi, Phys. Rev. B 53, 16338 (1996).

${ }^{27}$ O. Madelung, Semiconductors: Data Handbook, 3rd ed. (Springer, Berlin, 2004)

${ }^{28}$ L. M. Landsberger and W. A. Tiller, Appl. Phys. Lett. 49, 143 (1986).

${ }^{29}$ G. D. Mahan, Many-Particle Physics, 2nd ed. (Plenum, New York, 1990).

${ }^{30}$ R. D. Schaller, J. M. Pietryga, and V. I. Klimov, Nano Lett. 7, 3469 (2007).

${ }^{31}$ J. D. Jackson, Classical Electrodynamics (Academic, New York, 1998). 